\title{
Formation of silica nanotubes through a TPPS J aggregates template
}

\author{
ZHANG Li $^{1 *},{\text { JIANG } \operatorname{Jian}^{2} \& \text { LIU MingHua }}^{1 *}$ \\ ${ }^{1}$ Beijing National Laboratory for Molecular Science, CAS Key Laboratory of Colloid, Interface and Chemical Thermodynamics, Institute of \\ Chemistry, Chinese Academy of Sciences, Beijing 100190, China; \\ ${ }^{2}$ National Center for Nanoscience and Technology, Beijing 100190, China
}

Received May 23, 2012; accepted July 3, 2012; published online September 12, 2012

\begin{abstract}
It is found that 5,10,15,20-tetrakis (4-sulfonatophenyl) porphyrin (TPPS), which is known to form J aggregates in water under low $\mathrm{pH}$ value, acts as a template for the hydrolyzing of organosilane. The nanotube structures of silica with 4-8 nm inner diameters are obtained, which is consistent with the height of TPPS J aggregates, indicating that TPPS J aggregates are indeed acting as the template for the sol-gel transcription. The TPPS J aggregation disappear when the medium $\mathrm{pH}$ value is shifted from acidic to basic, consequently formed silica nanostructures are amorphous nanosphere rather than nanofiber structures. In contrast, the copper phthalocyanine (TSCuPc), which does not exist as linear J aggregates, cannot induce the silica to form one dimensional nanofiber structures, implying that the templating effect of TPPS J aggregates for inorganic nanostructures.
\end{abstract}

J aggregates, sol-gel transcription, silica nanostructures

Citation: Zhang L, Jiang J, Liu M H. Formation of silica nanotubes through a TPPS J aggregates template. Chin Sci Bull, 2012, 57: 4322-4327, doi: 10.1007/ s11434-012-5432-4

Porphyrin aggregation has gained much attention for over half a century, owing to its interesting optical and electronic properties exhibited by its self-assembled nanostructures [1-3]. In this field, a water-soluble tetrakis(4-sulfonatophenyl) porphyrin, TPPS, is well studied, because its diacid species can form $\mathrm{J}$ aggregates under aqueous conditions [4-8], high ionic strength, and can be induced by other cationic species including polypeptides [9-12], ionic liquid [13,14], surfactant and polyelectrolyte [15]. At acid aqueous, the TPPS exists as a zwitterionic state with peripheral $\mathrm{SO}_{3}^{2-}$ groups and cationic pyrrole ring resulting from the protonation of the pyrrole nitrogen. Then the $\pi-\pi$ stacking between overlapping phenyl rings and electrostatic interactions between the negative sulfonate groups and the protonated pyrrole nitrogen atoms are the main driving force for the $\mathrm{J}$ aggregation of TPPS. The formation of TPPS $\mathrm{J}$ aggregates is usually characterized by a sharp and intense absorbance at 490 $\mathrm{nm}$ (J-band) which shows a large red shift relative to the monomer absorption at about $434 \mathrm{~nm}$ (the soret band). Corresponding Q-band at about 600-700 nm also shows red

*Corresponding authors (email: liumh@iccas.ac.cn; zhangli@iccas.ac.cn) shifted and enhancement in intensity relative to the monomer as the result of aggregation of $\mathrm{H}_{2}$ TSPP [2-16]. A great deal of techniques, including RLS [17], small-angle X-ray scattering (SAXS) [18], atomic force microscopy (AFM) [19], and scanning tunneling microscopy (STM) [20] has proved aggregates form nanorod structures where the individual TPPS molecules are arranged in a head-to tail fashion.

Thus, the linear aggregates are the suitable candidate for the synthesis of some fibriform polymer or inorganic structures. For example, Shinkai reported that the assembly of TPPS acts as a template for electropolymerization of aniline, EDOT and pyrrole on the ITO electrode [21-23]. The corresponding monomers grow up to a nanosized rod-like structure $(30-50 \mathrm{~nm}$ in diameter and several hundred nanometers in length), reflecting the aggregation mode of TPPS. In addition, TPPS supermolecules can act as a template to transcribe its organic superstructures into inorganic materials by a sol-gel reaction of metal alkoxides (sol-gel transcription), by which one can control the morphology of inorganic compounds and create various new superstructural inorganic materials. Che transcribed the organic structure into chiral mesostructured porphyrin-silica hybrid using a 
cationic organolsilane by a sol-gel reaction [24]. She used a cationic organosilane bearing a quaternary ammonium group to interact with anionic TPPS and then cohydrolyze with tetraethyl orthosilicate. Here, TPPS assembly was functioned as a template to prepare silica nanostructures. Since TPPS assemblies are very sensitive to the $\mathrm{pH}$, the silica nanostructure obtained by the sol-gel method may depend on the TPPS aggregates by the variation of $\mathrm{pH}$. In addition, in order to verify the template effect of TPPS J aggregates, we used $\mathrm{CuPc}$ as a reference, which cannot form $\mathrm{J}$ aggregates at the same condition. There was no fiber structure can be obtained when $\mathrm{CuPc}$ was used as a template, indicating that the silica fiber structures was transcribed from liner $\mathrm{J}$ aggregates.

\section{Materials and method}

\subsection{Materials}

Tetrakis(4-sulfonatophenyl)porphyrin (TPPS), was purchased from Dojindo Laboratories and used without further purification. 3,4,4,4-Tetrasulfonic acid tetrasodium salt copper phthalocyanine (TS-CuPc) was used as received. The scheme of zwitterionic TPPS and CuPc are shown in Scheme 1. Hydrochloride and $\mathrm{NaOH}$ were used to adjust the $\mathrm{pH}$ value of TPPS or CuPC solution. 3-Aminopropyltriethoxysilane (APES) and tetraethyl orthosilicate (TEOS) were purchased from Alfa Co. The molecular structures of TPPS, TS-CuPC, TEOS and APES are shown in Scheme 1.

\subsection{Preparation of $\mathrm{SiO}_{2}$ nanostructures through TPPS assembly as a template}

In the experiments for preparing $\mathrm{SiO}_{2}$ nanostructures, TPPS solutions were prepared using Millipore water $(18 \mathrm{M} \Omega \mathrm{cm})$ and the $\mathrm{pH}$ was adjusted by hydrochloric acid and $\mathrm{NaOH}$. The APES and TEOS in which the ratio is kept at $1: 10$ were added into TPPS solution and further vigorous stirring for $3 \mathrm{~h}$. The mixture was incubated at room temperature for $5 \mathrm{~d}$. And then the mixtures were collected by centrifugal separation and washed with water at least five times in order to remove TPPS.

\subsection{Characterization}

The absorption spectra were obtained from a JASCO UV550 spectrophotometer. SEM (scanning electron microscope) was performed using a Hitachi S-4800 system with an accelerating voltage of $15 \mathrm{kV}$. Samples for SEM were prepared by cast the nanostructure aqueous dispersion on silicon slices. TEM (transmission electron microscope) images were obtained on a JEM-100CX II electron microscope operating at an accelerating voltage of $100 \mathrm{kV}$. EDX was used to measure the elemental analysis of $\mathrm{TiO}_{2} / \mathrm{SiO}_{2}$ composite. The TEM samples were prepared by placing a small amount of silica nanostructure dispersion on carbon-coated copper grids (300 mesh).

\section{Results and discussion}

\section{1 $\mathrm{SiO}_{2}$ nanostructures obtained by TPPS template}

The protonation of the pyrrole nitrogen takes place upon decreasing $\mathrm{pH}$ below about 5 , while the sulfonic acid groups remain unprotonated until much lower $\mathrm{pH}$ [25]. Then at $\mathrm{pH}$ $3\left(1 \times 10^{-5} \mathrm{~mol} / \mathrm{L}\right)$, the TPPS exist as a zwitterionic species, characterized by the Soret and Q bands at 434 and $644 \mathrm{~nm}$, respectively, as shown in Figure 1. These bands show

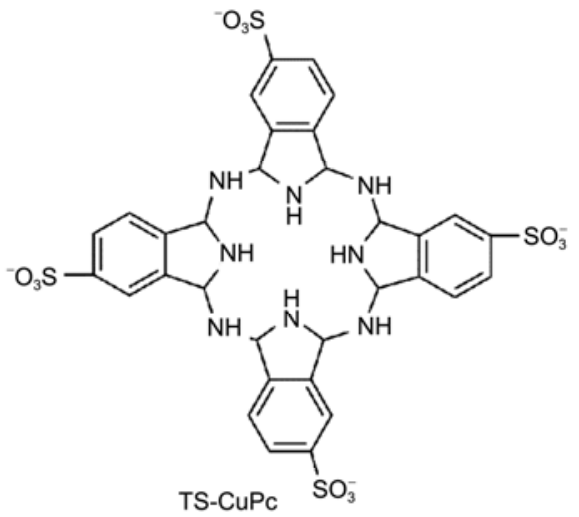

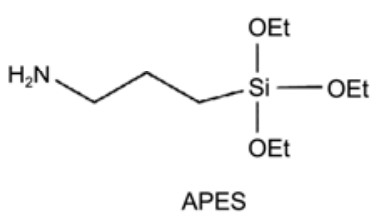

Scheme 1 Structures of TPPS, TS-CuPc, TEOS and APES. 


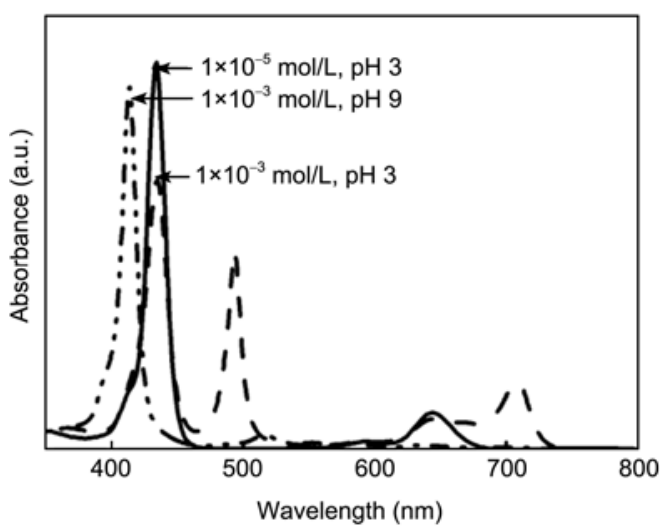

Figure 1 UV-Vis absorption spectra of TPPS solution at different $\mathrm{pH}$ : The 490 and $704 \mathrm{~nm}$ absorbances are assigned to the J aggregates transition. The $1.0 \mathrm{~cm}$ and $1.0 \mathrm{~mm}$ path length cell were used for the measurement of TPPS $\left(1 \times 10^{-5} \mathrm{~mol} / \mathrm{L}\right)$ and $\left(1 \times 10^{-3} \mathrm{~mol} / \mathrm{L}\right)$, respectively.

red-shift relative to the adsorption band of free TPPS (414 $\mathrm{nm})$. Upon increase the concentration to $1 \times 10^{-3} \mathrm{~mol} / \mathrm{L}$, the new bands appeared at 491 and $702 \mathrm{~nm}$, respectively, indicating the formation of TPPS $\mathbf{J}$ aggregates. When the $\mathrm{pH}$ value is above 7, the soret band shifts to $414 \mathrm{~nm}$, accompanying the disappearance of adsorption band at 490 and 702 $\mathrm{nm}$, indicating the TPPS exists as a free base monomer with a net -4 charge. In the free base TPPS, peripheral phenyl rings are perpendicular to the plane of the porphyrin ring, while the protonation of the pyrrole nitrogen at lower $\mathrm{pH}$ value causes phenyl groups are closer to being in plane with the porphyrin ring. The change in conformation of phenyl group causes the blue-shift of adsorption band from zwitterionic species to free base, and also disturb the disassembly of TPPS aggregates.

The APES with equal molar amount to TPPS and ten times TEOS was added into TPPS solution. After subsequent stirring for $3 \mathrm{~h}$, the mixture was kept at room temperature for $5 \mathrm{~d}$. Figure 2(a) shows a SEM image of the formed silica nanostructures in the presence of $1 \times 10^{-5} \mathrm{~mol} / \mathrm{L}$ TPPS (pH 3). Some amorphous nanosphere structures can be observed. In the case of $1 \times 10^{-3} \mathrm{~mol} / \mathrm{L}$ TPPS at $\mathrm{pH} 7$ (Figure 2(b)), the similar nanosphere structures with the radius of 400-500 nm can be obtained. When the system $\mathrm{pH}$ value decrease to 3 (Figure 2(c)), some intertwined nanofiber structures with $20-30 \mathrm{~nm}$ in width and several to tens of micrometers in length appears.

Since the APES used here is a kind of weak base, which can influence TPPS assembly, we explored that the TPPS aggregates in the presence of variation of APES concentration and the corresponding silica nanostructures. Figure 3 shows that the UV spectra of TPPS $\left(1 \times 10^{-3} \mathrm{~mol} / \mathrm{L}, \mathrm{pH} 3\right)$ upon variation of APES concentration. As we mentioned above, the TPPS exists as $\mathbf{J}$ aggregates at this condition. Upon addition of APES into the TPPS solution ( $\mathrm{pH} \mathrm{3}$, $1 \times 10^{-3} \mathrm{~mol} / \mathrm{L}$ ), when the ratio of APES to TPPS below 2, the $\mathrm{J}$ aggregates still exist, characterized by the TPPS
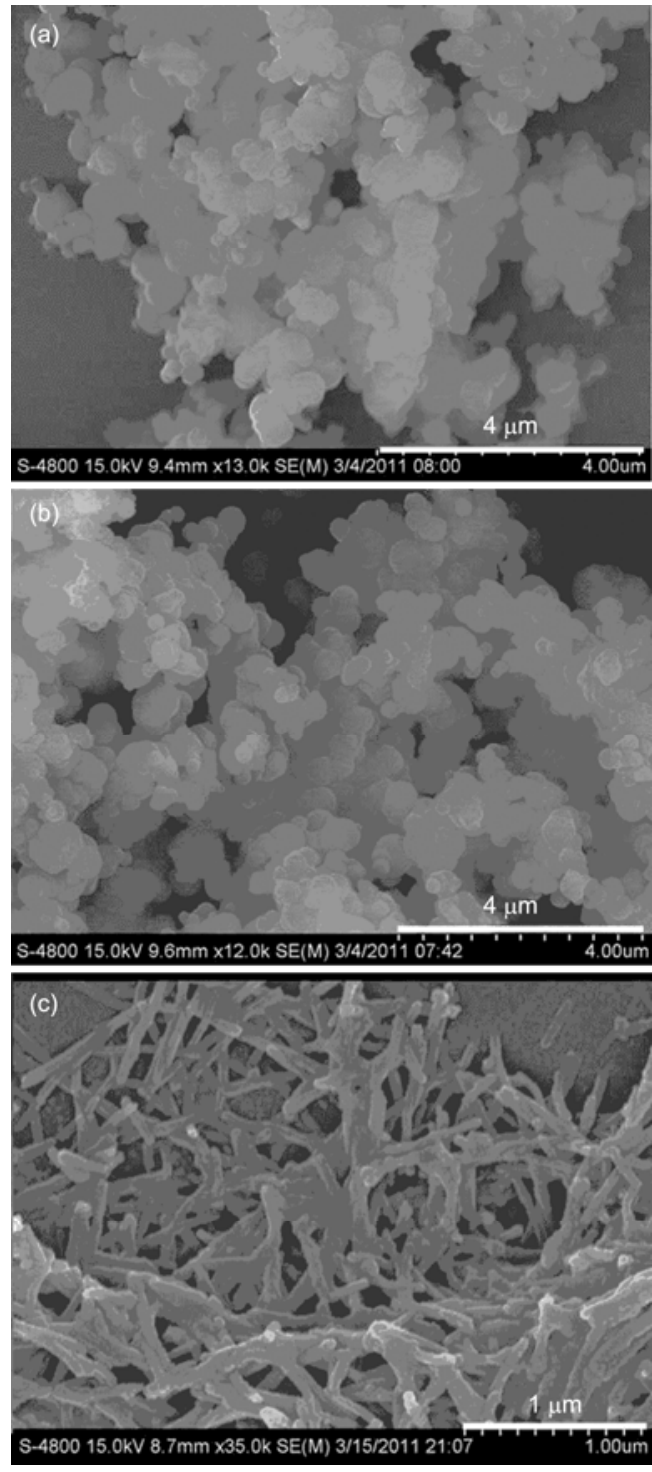

Figure 2 The formed silica nanostrucures in the presence of TPPS (a) $1 \times 10^{-5} \mathrm{~mol} / \mathrm{L}$, pH 3 ; (b) $1 \times 10^{-3} \mathrm{~mol} / \mathrm{L}, \mathrm{pH} 7$ and (c) $1 \times 10^{-3} \mathrm{~mol} / \mathrm{L}, \mathrm{pH} 3$.

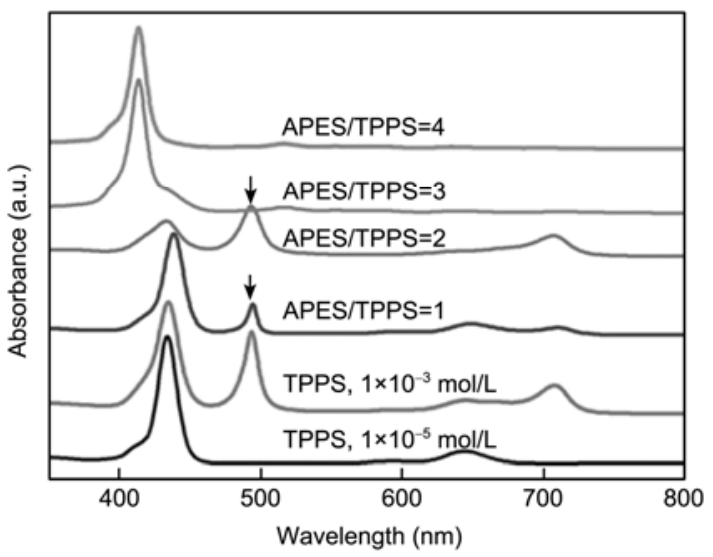

Figure 3 UV-Vis spectra of TPPS at variation of APES. A $1.0 \mathrm{~cm}$ and $1.0 \mathrm{~mm}$ path length cell were used for the measurement of TPPS $\left(1 \times 10^{-5}\right.$ $\mathrm{mol} / \mathrm{L})$ and $\left(1 \times 10^{-3} \mathrm{~mol} / \mathrm{L}\right)$, respectively. 
J-band at $490 \mathrm{~nm}$. While the ratio of APES increase to 3 and 4 , the $\mathrm{J}$ aggregates of TPPS were changed to free base species as the result of the unprotonation of pyrole $\mathrm{NH}$ by $\mathrm{NH}_{2}$ group of APES. The color of TPPS changed from green to dark purple with the increase concentration of APES, indicating the unprotonation of TPPS.

Figure 4 shows that the morphology of silica nanostructures induced by TPPS assembly at variation APES ratio.
The nanofiber structures of TPPS $\mathbf{J}$ aggregates are transcribed into the silica nanostructure (Figure 4(a) and (b)). The nanosphere structures are obtained when the ratio of APES increased to 3, in which the TPPS exists as free base monomers. TEM revealed that the silica nanofiber structures are actually composed nanotubes, and the inner diameters are within 4-8 nm, as shown in Figure 5(a) and (b). TPPS J aggregates morphology was ever imaged by AFM

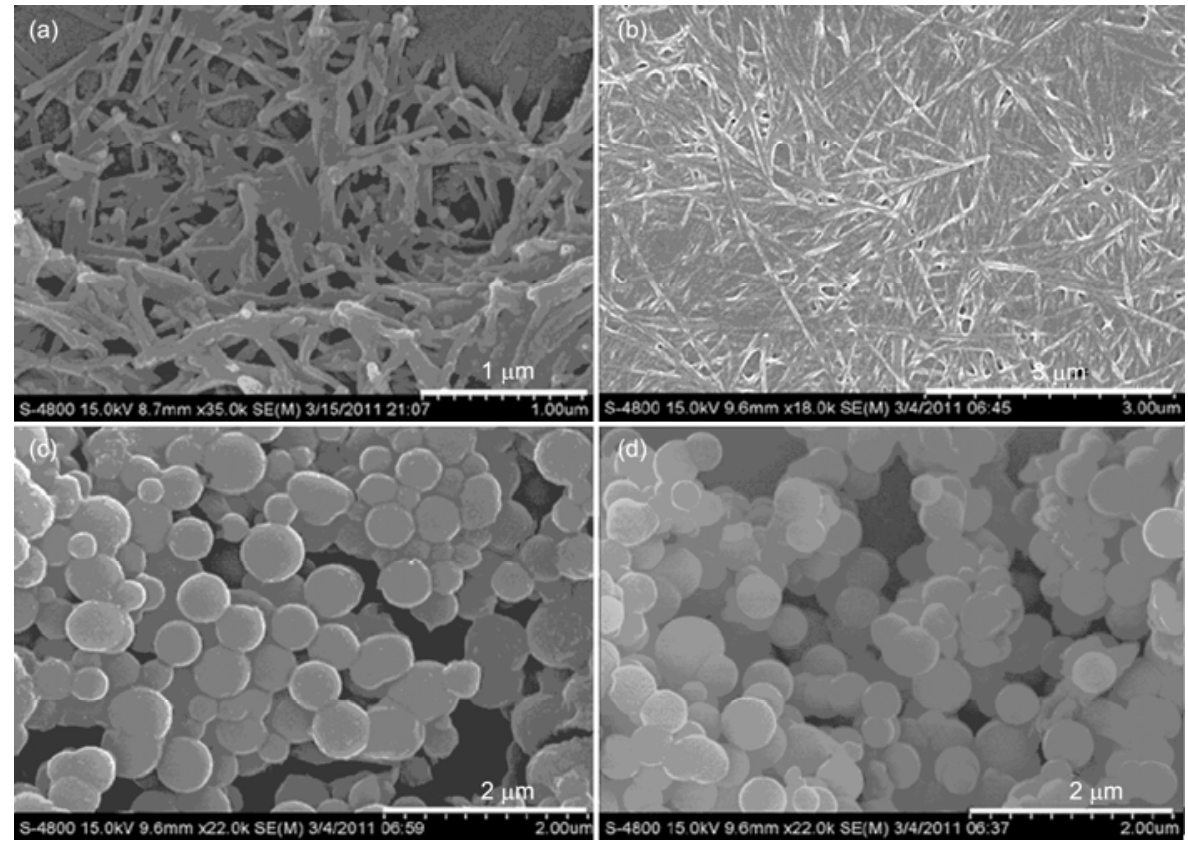

Figure 4 SEM images of formed silica nanostructures in the presence of TPPS $\left(1 \times 10^{-3} \mathrm{~mol} / \mathrm{L}, \mathrm{pH} 3\right)$ at variation of APES to TPPS ratio (a) 1 ; (b) 2 ; (c) 3 and (d) 4.
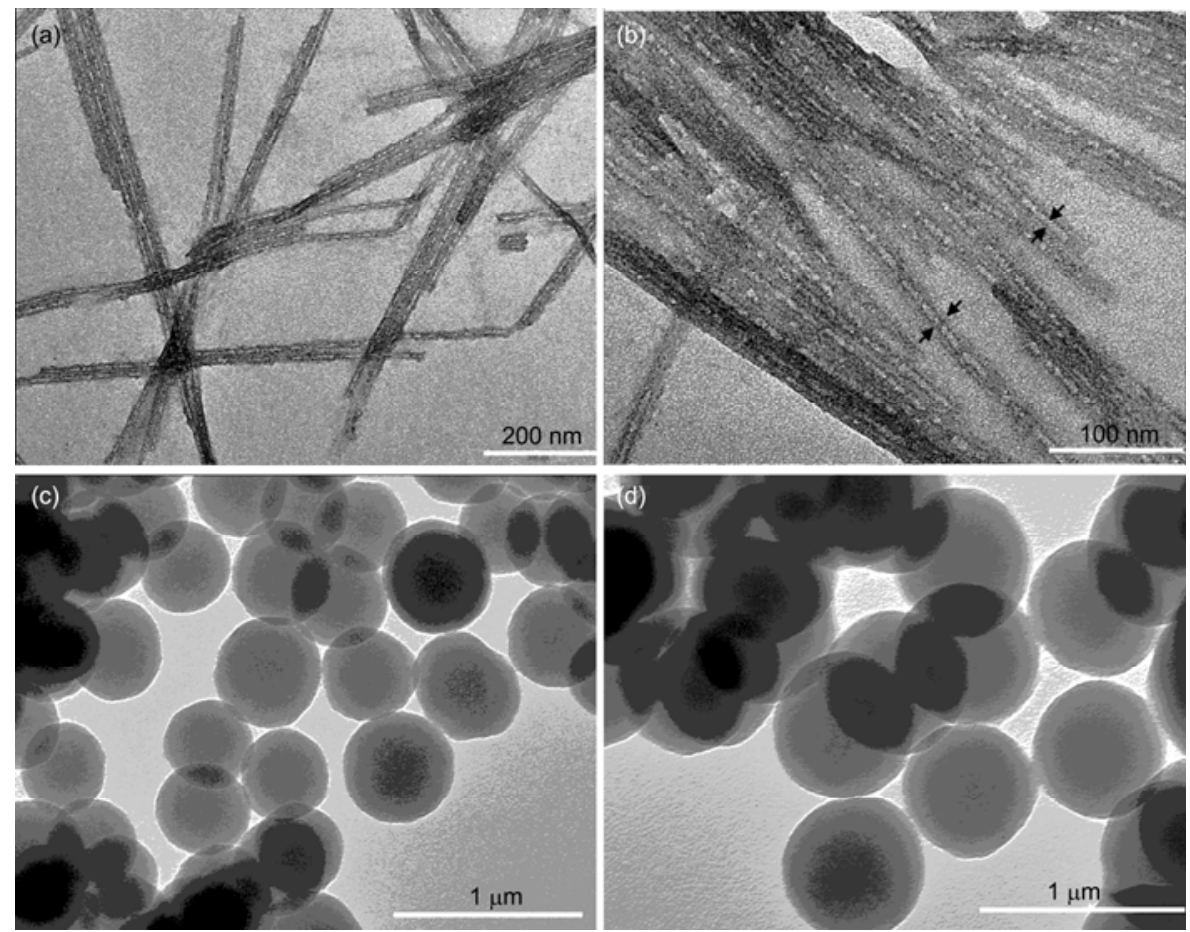

Figure 5 TEM images of formed silica nanostrucures in the presence of TPPS $\left(1 \times 10^{-3} \mathrm{~mol} / \mathrm{L}, \mathrm{pH} 3\right)$ at variation of APES to TPPS ratio (a) 1 ; (b) 2 ; (c) 3 and (d) 4. 


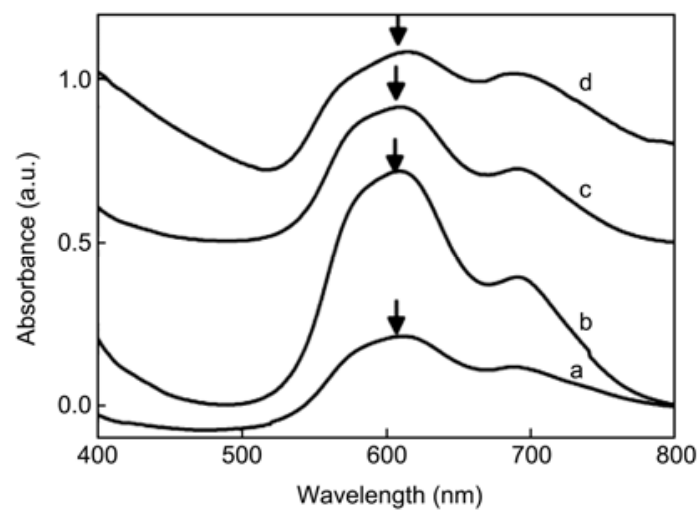

Figure 6 UV-Vis spectra of TS-CuPc (a) dilute solution $\left(1 \times 10^{-6} \mathrm{~mol} / \mathrm{L}\right)$; (b) concentrated solution $\left(1 \times 10^{-3} \mathrm{~mol} / \mathrm{L}\right)$; (c) $1 \times 10^{-3} \mathrm{~mol} / \mathrm{L}$ TS-CuPc solution $\left(\mathrm{pH} 3\right.$ ) with APES (the ratio of APES to TS-CuPc is 1) and (d) $1 \times 10^{-3} \mathrm{~mol} / \mathrm{L}$ TS-CuPc solution ( $\mathrm{pH} 3$ ) with APES (the ratio of APES to TS-CuPc is 4).

using mica, HOPG or Au(111) as substrates, and the nanorod structures with $18-40 \mathrm{~nm}$ for the width and $4-10 \mathrm{~nm}$ for the height were reported in the literature [20]. The height of nanorod is consistent with the inner diameter of silica nanotube, indicating that the TPPS J aggregates were indeed acted as the template for the hydrophisis. In the case of the ratio of APES to TPPS increasing to 3 and 4 , the $\mathrm{NH}_{2}$ moiety of APES resulted in the unprotonization and the finally the disassembly of $\mathbf{J}$ aggregates, consequently the silica nanotube structures disappeared. Some solid nanosphere structures with the diameter around 400-500 nm was obtained. This result further reveals that the linear $\mathrm{J}$ aggregates of TPPS can be transcribed into inorganic structure by sol-gel method.

\section{2 $\mathrm{SiO}_{2}$ nanostructures obtained in the presence of CuPc}

In order to further prove the templating effect of TPPS $\mathbf{J}$ aggregates for inorganic nanotube structures, the $\mathrm{CuPc}$ was used as a reference. Figure 6 shows that UV-Vis spectrum of CuPc and the complex of CuPc with APES/TEOS. Spectrum a in Figure 6 shows the visible absorption spectra of a dilute aqueous solution of TS-CuPc $\left(1 \times 10^{-6} \mathrm{~mol} / \mathrm{L}\right)$. The large peak in the region 550-700 $\mathrm{nm}$ corresponds to a Q-band. The Q-band in the dilute aqueous solution is split in two, 671 and $610 \mathrm{~nm}$, which can be ascribed to the monomer absorption $(671 \mathrm{~nm})$ and aggregate adsorption $(610 \mathrm{~nm})$. The latter can be attributed to absorption by dimmers or higher aggregates. The peak at $671 \mathrm{~nm}$ corresponds to the amorphous state in which adjacent molecules adopt no particular orientation with respect to one another. Spectrum $b$ in Figure 6 corresponds to the concentrated solution of TS-CuPc $\left(C=1 \times 10^{-3} \mathrm{~mol} / \mathrm{L}, \mathrm{pH} 3\right)$. The adsorption band shows a dominant maximum at $610 \mathrm{~nm}$ and a shoulder peak at $690 \mathrm{~nm}$. This suggests that the peak at lower wavelengths corresponds to molecular aggregates, cofacially stacked dimmers and oligomers [26,27]. When APES and TEOS were added into the TS-CuPc solution, the shape of spectrum was similar with the absence of APES and TEOS, with the relative intensity adsorption of cofacially stacked aggregates
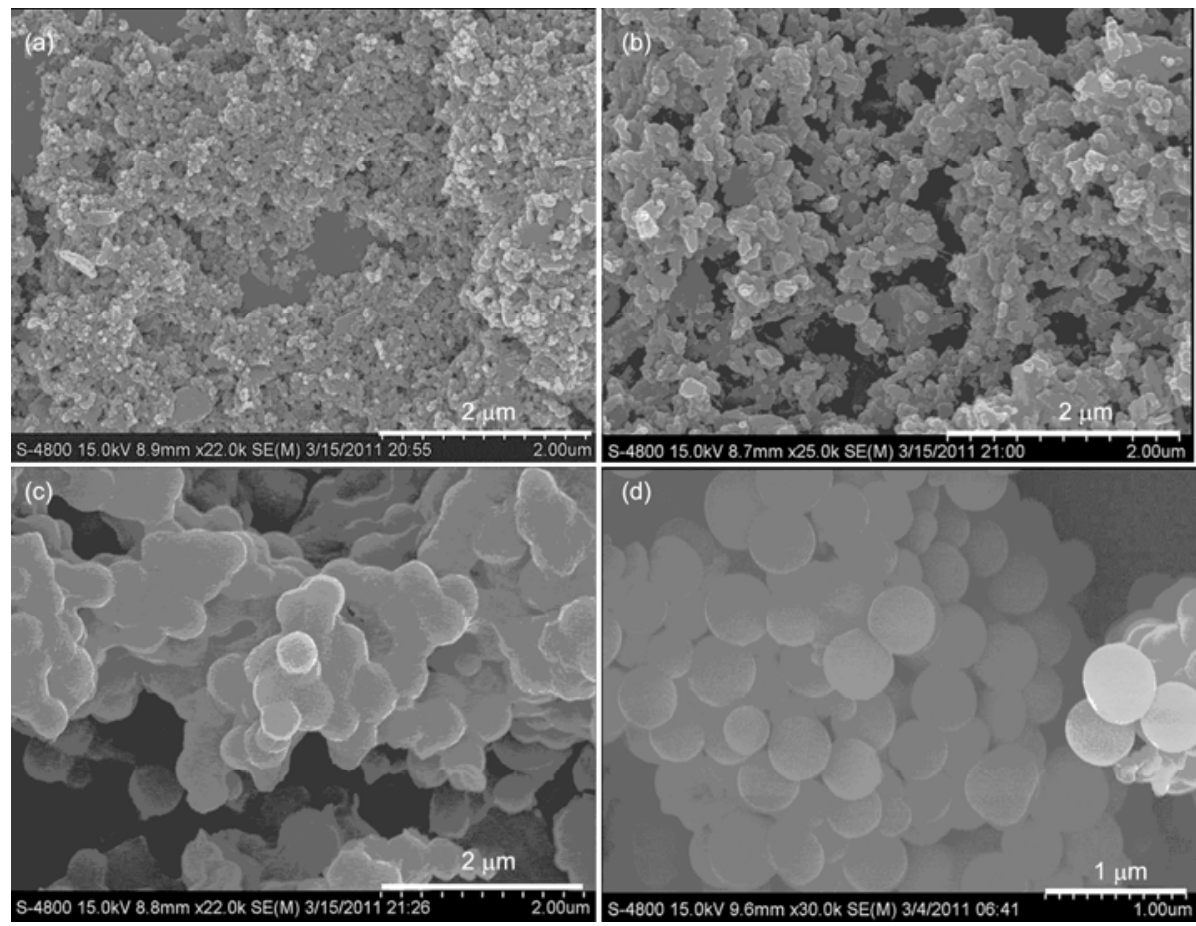

Figure 7 SEM images of formed silica nanostrucures in the presence of TS-CuPc $\left(1 \times 10^{-3} \mathrm{~mol} / \mathrm{L}\right.$, $\mathrm{pH} 3$ ) at variation of APES to TS-CuPc ratio (a) 1; (b) 2; (c) 3 and (d) 4 
slightly lower. These results indicate that TS-CuPc cannot form $\mathrm{J}$ aggregates at aqueous solution and in the presence of APES. When the hydrolysis of APES and TEOS was executed at $\mathrm{CuPC}$ solution, the nanosphere structures can be obtained at the ratio of APES to TPPS from 1 to 4 . In other words, there is no one dimensional nanofiber structures can be obtained, support that the linear structure of $\mathrm{J}$ aggregates playing an important role in the synthesis silica nanofiber structures.

\section{Conclusions}

TPPS J aggregates are important supramolecular system, and here we have demonstrated that they can act as the template to prepare silica nanostructures. The SEM and TEM images showed that the nanotube structures only can be obtained in the presence of $\mathbf{J}$ aggregates. And the inner diameter of the nanotube is consistence with the height of nanorod of TPPS $\mathbf{J}$ aggregates revealed by AFM image, further indicating that the TPPS aggregates structures can be transcribed into inorganic structures. In contrast, the TS-CuPC, which does not form linear J aggregates, cannot induce the silica to form one dimensional nanofiber structures. Therefore, this is a novel transcription process of supramolecular templates to inorganic materials.

This work was supported by the National Basic Research Program of China (2009CB930802), the National Natural Science Foundation of China (91027042, 21021003), and the Fund of the Chinese Academy of Sciences.

1 Jeukens C, Lensen M C, Wijnen F, et al. Polarized absorption and emission of ordered self-assembled porphyrin rings. Nano Lett, 2004, 4: 1401-1406

2 Bonnett R. Photosensitizers of the porphyrin and phthalocyanine series for photodynamic therapy. Chem Soc Rev, 1995, 24: 19-33

3 Kano K. Molecular complexes of water-soluble porphyrins. J Porphyr Phthalocya, 2004, 8: 148-155

4 Ohno O, Kaizu Y, Kobayashi H. J-aggregate formation of a watersoluble porphyrin in acidic aqueous-media. J Chem Phys, 1993, 99: 4128-4139

5 Akins D L, Zhu H R, Guo C. Absorption and raman-scattering by aggregated meso-tetrakis( $p$-sulfonatophenyl)porphine. J Phys Chem, 1994, 98: 3612-3618

6 Akins D L, Ozcelik S, Zhu H R, et al. Fluorescence decay kinetics and structure of aggregated tetrakis( $p$-sulfonatophenyl)porphyrin. J Phys Chem, 1996, 100: 14390-14396

7 Akins D L, Zhu H R, Guo C. Aggregation of tetraaryl-substituted porphyrins in homogeneous solution. J Phys Chem, 1996, 100: 54205425
8 Pasternack R F, Schaefer K F, Hambright P. Resonance light-scattering-studies of porphyrin diacid aggregates. Inorg Chem, 1994, 33: 2062-2065

9 Bellacchio E, Lauceri R, Gurrieri S, et al. Template-imprinted chiral porphyrin aggregates. J Am Chem Soc, 1998, 120: 12353-12354

10 Purrello R, Bellacchio E, Gurrieri S, et al. $\mathrm{pH}$ modulation of porphyrins self-assembly onto polylysine. J Phys Chem B, 1998, 102: 8852-8857

11 Purrello R, Scolaro L M, Bellacchio E, et al. Chiral H- and J-type aggregates of meso-tetrakis(4-sulfonatophenyl)porphine on alpha-helical polyglutamic acid induced by cationic porphyrins. Inorg Chem, 1998, 37: 3647-3651

12 Koti A S R, Periasamy N. Self-assembly of template-directed J-aggregates of porphyrin. Chem Mater, 2003, 15: 369-371

$13 \mathrm{Wu} \mathrm{J} \mathrm{J,} \mathrm{Li} \mathrm{N,} \mathrm{Li} \mathrm{K} \mathrm{A,} \mathrm{et} \mathrm{al.} \mathrm{J-aggregates} \mathrm{of} \mathrm{diprotonated} \mathrm{tetrakis}$ (4-sulfonatophenyl)porphyrin induced by ionic liquid 1-butyl-3methylimidazolium tetrafluoroborate. J Phys Chem B, 2008, 112: 8134-8138

14 Zhang L, Tian Y, Liu M. Ionic liquid induced spontaneous symmetry breaking: Emergence of predominant handedness during the selfassembly of tetrakis(4-sulfonatophenyl)porphyrin (TPPS) with achiral ionic liquid. Phys Chem Chem Phys, 2011, 13: 17205-17209

15 Zhao L, Wang X, Li Y, et al. Chiral micelles of achiral TPPS and diblock copolymer induced by amino acids. Macromolecules, 2009, 42: 6253-6260

16 Shelnutt J A. A simple interpretation of raman excitation-spectra of metalloporphyrins. J Chem Phys, 1981, 74: 6644-6657

17 Chen X D, Liu M H. Induced chirality of binary aggregates of oppositely charged water-soluble porphyrins on DNA matrix. J Inorg Biochem, 2003, 94: 106-113

18 Gandini S C M, Gelamo E L, Itri R, et al. Small angle X-ray scattering study of meso-tetrakis (4-sulfonatophenyl) porphyrin in aqueous solution: A self-aggregation model. Biophys J, 2003, 85: 1259-1268

19 Schwab A D, Smith D E, Bond-Watts B, et al. Photoconductivity of self-assembled porphyrin nanorods. Nano Lett, 2004, 4: 1261-1265

20 Friesen B A, Nishida K R A, McHale J L, et al. New nanoscale insights into the internal structure of tetrakis(4-sulfonatophenyl) porphyrin nanorods. J Phys Chem C, 2009, 113: 1709-1718

21 Hatano T, Bae A H, Takeuchi M, et al. New morphology-controlled poly(aniline) synthesis using anionic porphyrin aggregate as a template and proton-driven structural changes in the porphyrin aggregate. B Chem Soc Jpn, 2004, 77: 1951-1957

22 Hatano T, Takeuchi M, Ikeda A, et al. Nano-rod structure of poly (ethylenedioxythiophene) and poly(pyrrole) as created by electrochemical polymerization using anionic porphyrin aggregates as template. Org Lett, 2003, 5: 1395-1398

23 Hatano T, Takeuchi M, Ikeda A, et al. New morphology-controlled poly(aniline) synthesis using anionic porphyrin aggregate as a template. Chem Lett, 2003, 32: 314-315

24 Qiu H B, Xie J J, Che S A. Formation of chiral mesostructured porphyrin-silica hybrids. Chem Commun, 2011, 47: 2607-2609

25 Kalyanasundaram K. In Photochemistry of Polypyridine and Porphyrin Complexes. London: Academic Press, 1992. 428

26 Schumann S, Hatton R A, Jones T S. Organic photovoltaic: Devices based on water-soluble copper phthalocyanine. J Phys Chem C, 2011, 115: 4916-4921

27 Hatton R A, Blanchard N P, Stolojan V, et al. Nanostructured copper phthalocyanine-sensitized multiwall carbon nanotube films. Langmuir, 2007, 23: 6424-6430

Open Access This article is distributed under the terms of the Creative Commons Attribution License which permits any use, distribution, and reproduction in any medium, provided the original author(s) and source are credited. 